\title{
All-optical preparation of molecular ions in the rovibrational ground state
}

\author{
T. Schneider ${ }^{\star}$, B. Roth, H. Duncker, I. Ernsting and S. Schiller ${ }^{\star}$
}

In the field of cold quantum matter, control of the motional degrees of freedom of both neutral and charged gas-phase molecules has been achieved for a wide range of species ${ }^{1-11}$. However, cooling of the internal degrees of freedom remains challenging. Recently, transfer to the internal ground state by sophisticated optical techniques has been demonstrated for neutral alkali dimers created in single quantum states from ultracold atoms ${ }^{12-15}$. Here we demonstrate cooling of the rotational degree of freedom of heteronuclear diatomic molecules with a thermal distribution of internal states, using a simple, robust and general optical-pumping scheme with two low-power continuous-wave lasers. With trapped and translationally cooled hydrogen deuteride $\left(\mathrm{HD}^{+}\right)$molecular ions as a

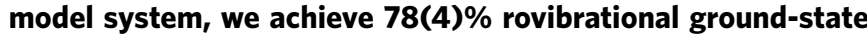
population. The rotationally, vibrationally and translationally cold molecular ion ensemble is suitable for a number of applications, such as generation of long-lived coherences or frequency metrology of fundamental constants ${ }^{16,17}$.

The study of cold molecular systems promises new insights and advances in many fields of physics and physical chemistry. As in atomic physics, the key to tapping the full potential of molecules is the ability to accurately control the external and internal degrees of freedom of the particles. The complex internal structure of molecules has however so far precluded direct application of many techniques developed for trapping and cooling of atoms, demanding modified or completely new approaches. Now, a large toolbox for trapping and cooling the motional degrees of freedom of both neutral and charged molecules is available ${ }^{18}$. Although general schemes for cooling the internal degrees of freedom of molecules have been proposed ${ }^{19,20}$, the most general method available at present is cryogenic buffer-gas cooling, which is efficient only for molecules in the vibrational ground state and limits the translational temperature to a few hundred millikelvin ${ }^{7}$. Coherent transfer to the rovibrational ground state $e^{11-14}$ is most suitable when most of the molecules are initially in the same quantum state, as is the case for molecules produced by associating cold atoms.

For heteronuclear molecular ensembles for which the population is distributed among many rotational levels in the $v=0$ vibrational manifold, optical pumping has been proposed as an approach to rotational cooling ${ }^{21,22}$. We demonstrate here that a scheme using two laser fields driving a fundamental and an overtone vibrational electric dipole transition ${ }^{21}$ yields a large ground-state population and briefly discuss the applicability of the scheme to various diatomic molecular species.

Figure 1 shows the energy levels (without hyperfine structure) and electric dipole transitions of the $\mathrm{HD}^{+}$molecule relevant for the experiment. The initial internal-state distribution is given by a Boltzmann distribution reflecting thermal equilibrium with the $T \cong 300 \mathrm{~K}$ blackbody radiation field emitted by the experimental

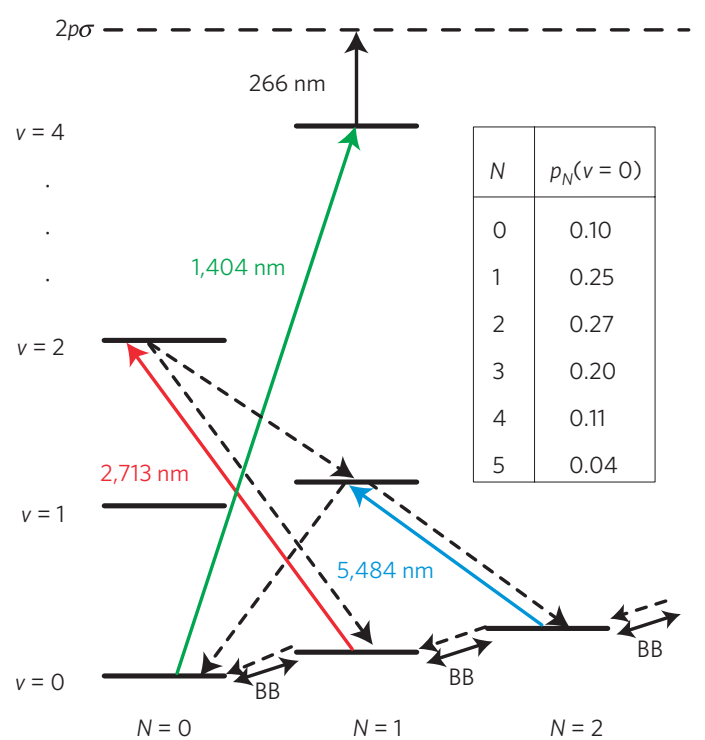

Figure 1 | Schematic view of relevant rovibrational states and dipole transitions in the $1 s \sigma$ electronic ground state of HD $^{+}$(not to scale). 5,484- and 2,713-nm transitions: optical pumping. 1,404- and 266-nm transitions: state-selective detection by $\left(1+1^{\prime}\right)$-photon dissociation. BB: blackbody radiation. Dashed lines: dipole spontaneous decay channels. $p_{N}$ : theoretical fractional rotational state populations in $v=0$ at $T=300 \mathrm{~K}$.

apparatus. For $\mathrm{HD}^{+}$, this means that all of the ions are in the $v=0$ vibrational state of the $1 s \sigma$ electronic ground state, but only $10 \%$ are in the $(v=0, N=0)$ absolute rovibrational ground state. The remaining population is distributed mostly among the states $(v=0, N=1-5)$ with a maximum of $27 \%$ residing in the $(v=0, N=2) \operatorname{state}^{23}$.

The set $\{(0,0),(0,1),(0,2),(1,1),(2,0)\}$ of rovibrational states $(v, N)$ forms a nearly closed five-level system with the rovibrational ground state as a dark state when interacting with two laser fields driving the $(0,1) \rightarrow(2,0)$ and $(0,2) \rightarrow(1,1)$ transitions. This leads to rapid optical pumping of the populations in $(0, N=1,2)$ to the $(0,0)$ ground state. If the optical pump rates sufficiently exceed the rates at which the blackbody radiation redistributes the populations among rotational states, also most of the population in $(0, N>2)$ states is transferred to the rovibrational ground state on a timescale given approximately by the inverse of the average blackbody redistribution rate. As the spontaneous emission rates from the excited $(2,0)$ and $(1,1)$ states are large $\left(32 \mathrm{~s}^{-1}, 18 \mathrm{~s}^{-1}\right)$ compared with the blackbody redistribution rates $\left(<1 \mathrm{~s}^{-1}\right)$, those conditions can be easily met and also redistribution among excited states by blackbody radiation is negligible. The 


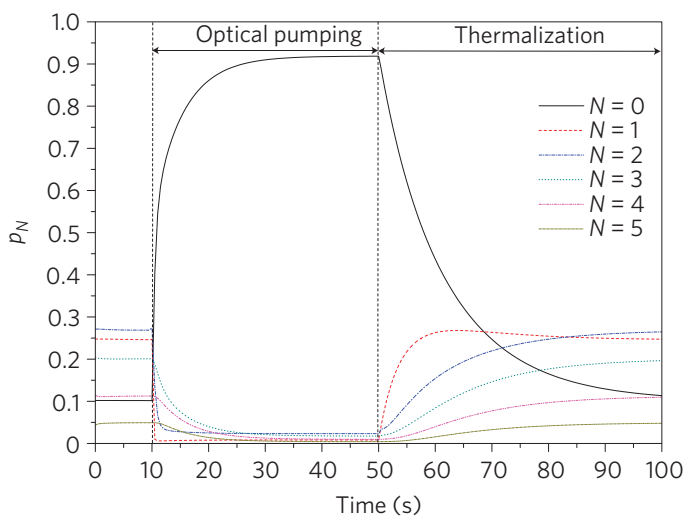

Figure 2 | Rate equation simulation for the optical pumping scheme. The time evolution of the fractional rotational state populations $p_{N}$ in the vibrational ground state for simultaneous optical pumping on the $(0,1) \rightarrow(2,0)$ and $(0,2) \rightarrow(1,1)$ transitions as well as thermalization with the $T=300 \mathrm{~K}$ blackbody radiation after the optical pumping fields have been turned off.

maximum achievable steady-state population in the ground state is then limited by thermal excitation away from the ground state on the $(0,0) \rightarrow(0,1)$ transition, with rate $\Gamma_{00,01}(T) \cong 0.086 \mathrm{~s}^{-1}$.

We model the internal state dynamics by a set of rate equations, see the Methods section. Under optimum conditions an accumulation $p_{0} \cong 92 \%$ of the population in the rovibrational ground state is predicted to be reached within $40 \mathrm{~s}$ (see Fig. 2). Driving only the $(0,2) \rightarrow(1,1)$ transition, $p_{0} \cong 69 \%$ is predicted. The steady-state population fraction $1-p_{0}$ not residing in the ground state is predicted to scale as $\Gamma_{00,01}(T) / A_{\text {vib }}$, where $A_{\text {vib }}$ is the spontaneous emission rate for a vibrational decay $\Delta v=1$ (see Supplementary Information for an explicit expression for $p_{0}$ ). We expect that the two-laser optical pumping scheme can be applied also to many other heteronuclear diatomic molecules with comparable efficiency and simplicity because for many heteronuclear molecular ions $\Gamma_{00,01}(T) / A_{\text {vib }} \ll 1$ at room temperature (here, 0.015 ). Numerical simulations done for various diatomics with different electronic ground-state configurations predict state populations $>80 \%$ to be reachable within a few minutes also for molecules that have moderately smaller rotational constants than $\mathrm{HD}^{+}$(refs 21, 24 and Supplementary Information). For molecules with small rotational constants, a more sophisticated approach could yield similar results (see Supplementary Information).

When optical pumping is stopped, the ground-state population $p_{0}$ decays exponentially with a time constant $\tau \cong \Gamma_{00,01}(T)^{-1} \cong 11 \mathrm{~s}$ as the system evolves back to thermal equilibrium. During the first $100 \mathrm{~ms}, p_{0}$ changes by less than $1 \%$. For most types of spectroscopic and optical measurement, this is sufficient time for any further desired manipulation of the ground-state molecules.

We apply the optical state-preparation scheme to ensembles of typically $100 \mathrm{HD}^{+}$ions trapped together with about 2,000 laser-cooled atomic $\mathrm{Be}^{+}$ions in a linear radiofrequency quadrupole ion trap under conditions where the ions form a Coulomb crystal (for details of the experimental set-up, see the Methods section and refs 9, 23). The laser-cooled $\mathrm{Be}^{+}$ions provide sympathetic translational cooling for the $\mathrm{HD}^{+}$ions to a (secular) temperature of about $50 \mathrm{mK}$ and a means for detection of the molecules (see below).

The experimental sequence is shown in Fig. 3. We first load $\mathrm{HD}^{+}$ions into the trap. We resonantly excite the $\mathrm{HD}^{+}$ions' secular motion for $t_{\mathrm{s}}=5 \mathrm{~s}$ with an auxiliary radiofrequency field and measure the average $\mathrm{Be}^{+}$fluorescence change $\left\langle\Delta \Gamma_{1}\right\rangle$ resulting from the extra heating of the Coulomb crystal with a heating rate $\Gamma_{n}$ proportional to the number of $\mathrm{HD}^{+}$ions in the trap $n$. The total

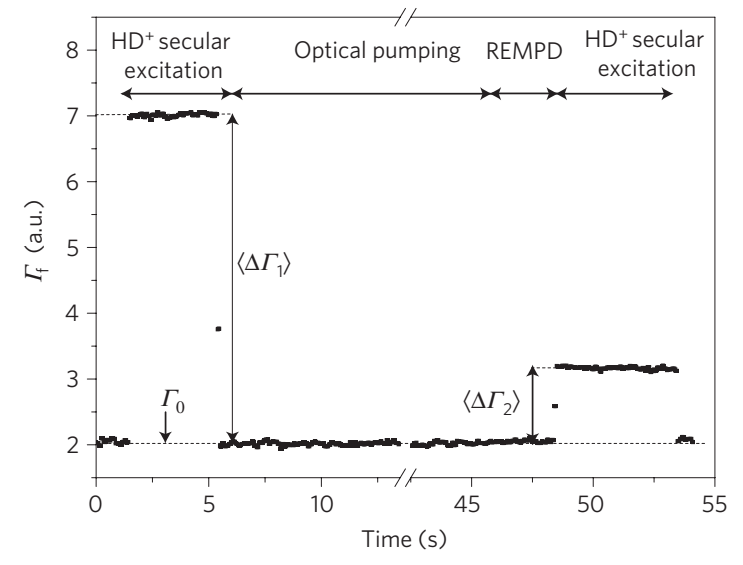

Figure 3 | Experimental sequence for determining the population $p_{N}$ in the state $(\boldsymbol{v}=\mathbf{0}, \boldsymbol{N})$. Typical experimental REMPD raw data for $N=0$. $\left\langle\Delta \Gamma_{1}\right\rangle\left(\left\langle\Delta \Gamma_{2}\right\rangle\right)$ is the average change in the $\mathrm{Be}^{+}$fluorescence $\Gamma_{\mathrm{f}}$ during the first (second) secular excitation.

heating rate is then $\Gamma_{\mathrm{h}}=\Gamma_{0}+\Gamma_{n}$, where $\Gamma_{0} \ll \Gamma_{n}$ is an approximately constant heating rate independent of $n$. Far away from the Doppler limit, laser cooling of the two-species Coulomb crystal can be described semiclassically in a similar way as for a single ion $^{25}$ and below saturation the cooling rate $\Gamma_{\mathrm{c}}$ is to good approximation proportional to the $\mathrm{Be}^{+}$fluorescence rate $\Gamma_{\mathrm{f}}$ (ref. 26). In the steady state, we have $\Gamma_{\mathrm{f}} \sim \Gamma_{0}+\Gamma_{n}$ with secular excitation and $\Gamma_{\mathrm{f}} \sim \Gamma_{0}$ without. We then optically pump with a quantum cascade laser at 5,484 $\mathrm{nm}$ and a distributed feedback diode laser at 2,713 nm (for details, see the Methods section) for $t_{\mathrm{p}}=40 \mathrm{~s}$ followed by $t_{\mathrm{d}}=3 \mathrm{~s}$ rotational state-selective $\left(1+1^{\prime}\right)$ resonance-enhanced multiphoton dissociation ${ }^{23}$ (REMPD): a continuous-wave ultraviolet laser at $266 \mathrm{~nm}\left(I_{\mathrm{UV}} \cong 128 \mathrm{~mW} \mathrm{~mm}^{-2}\right)$ dissociates molecular ions in the $v=4$ state through excitation to the $2 p \sigma$ excited state without affecting ions in the $v=0$ state. We can therefore selectively dissociate molecular ions from one specific $(0, N)$ state by simultaneous excitation of a $(0, N) \rightarrow(4, N \pm 1)$ transition with a broadly tunable continuous-wave diode laser $(1,370-1,480 \mathrm{~nm}$, $I_{\mathrm{IR}} \cong 19 \mathrm{~mW} \mathrm{~mm}^{-2}$ ) that is spectrally broadened to $285 \mathrm{MHz}$ fullwidth at half-maximum. Finally, we do another $5 \mathrm{~s}$ of secular excitation to obtain $\left\langle\Delta \Gamma_{2}\right\rangle$ and determine our detection signal

$$
S_{N}=\frac{\left\langle\Delta \Gamma_{1}\right\rangle-\left\langle\Delta \Gamma_{2}\right\rangle}{\left\langle\Delta \Gamma_{1}\right\rangle}=1-\frac{n_{2}}{n_{1}}
$$

where $n_{1}\left(n_{2}\right)$ is the initial (final) total number of $\mathrm{HD}^{+}$ions in the trap. For every state $(0, N=0-4)$, we repeat the sequence typically ten times and determine the average detection signal $\left\langle S_{N}\right\rangle$. The detection time was optimized by measuring $\left\langle S_{0}\right\rangle$ for successively increasing values of $t_{\mathrm{d}}$ until $\left\langle S_{0}\right\rangle$ starts to level off. If $t_{\mathrm{d}}$ is small compared with the blackbody thermalization time and spontaneous decay from the $(4, N \pm 1)$ state before dissociation can be neglected, the fractional population $p_{N}$ in the state $(0, N)$ is $p_{N}=S_{N}-S_{\mathrm{b}}$, where $S_{\mathrm{b}}$ is the background relative loss of $\mathrm{HD}^{+}$ions if the REMPD lasers are off during $t_{\mathrm{d}}$.

Figure 4 shows measurements of the rotational state distribution for $N=0-4$ with and without optical pumping together with the predictions of the rate equation calculations for our experimental parameters including the REMPD detection. For the optical pumping measurements, the background-corrected detection signals $\left\langle S_{N \mathrm{~b}}\right\rangle=\left\langle S_{N}\right\rangle-\left\langle S_{\mathrm{b}}\right\rangle\left(\left\langle S_{\mathrm{b}}\right\rangle=0.03(3)\right)$ are determined from 18 $(N=0), 10(N>0)$ and 25 (background) individual measurements each. For the ground state, we find $\left\langle S_{0 \mathrm{~b}}\right\rangle=0.78(4)$, which is $85 \%$ of the theoretical optimum $p_{0}=0.92$ and nearly a factor eight larger than the thermal equilibrium population at room temperature. The 


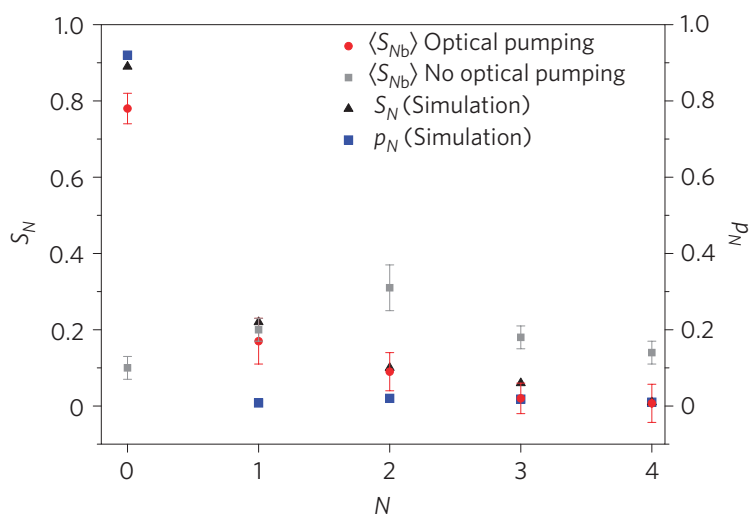

Figure 4 | Rotational-state distribution of the vibrational ground state after applying the optical pumping scheme. Red dots: mean background-corrected detection signals $\left\langle S_{N}\right\rangle-\left\langle S_{b}\right\rangle$. The error bars represent $1 \sigma$ standard deviation. Grey squares: measurement without optical pumping from ref. 23. Black triangles: detection signals predicted by rate equation simulations for the experimental parameters. Blue squares: fractional state populations $p_{N}$ predicted by rate equation simulations for the experimental parameters.

simulation predicts an actual detection signal $S_{0}=0.89$ limited by the available power of the ultraviolet-dissociation laser. The above value therefore represents a lower bound on the actual ground-state population. Comparing the ratios $R_{N}=\left\langle S_{N b}\right\rangle / S_{N}$ of measured to predicted detection signals for states with a sizable population $(N<3)$, we find values $R_{N}=0.8-0.9$. We also did measurements for variable optical pump times $t_{\mathrm{p}}$ as well as for optical pumping with only the quantum cascade laser $\left(\left\langle S_{0 b}\right\rangle=0.64(3)\right)$ or the distributed feedback diode laser $\left(\left\langle S_{0 \mathrm{~b}}\right\rangle=0.22(6)\right)$ where we again find $R_{N}=0.8-0.9$, confirming that the rate equation model presented above gives for the $\mathrm{HD}^{+}$molecule a good description of the internal-state dynamics. We ascribe the small discrepancy between theory and experiment to the simplified treatment of the hyperfine structure in the simulations combined with a lower than calculated excitation rate on the $(0, N) \rightarrow(4, N+1)$ transitions used for REMPD detection. The latter is evident from the unexpectedly long optimum detection time $t_{\mathrm{d}}=3 \mathrm{~s}$ found in the experiment, which can be explained only by a reduced excitation rate on the $(0, N) \rightarrow(4, N+1)$ transition during detection. Possible contributions to this effect are an imperfect overlap between the diode laser focus and the ion ensemble and the unnecessarily large and at present non-adjustable spectral broadening of the diode laser. The result is a reduction of $\mathrm{S}_{0}$ compared with the actual ground-state population $p_{0}$ because $t_{\mathrm{d}}$ is not sufficiently short compared with the blackbody thermalization time. For $N>0$, on the other hand, the simulations predict $S_{N}>p_{N}$ for the same reason, in good agreement with the experimental data. For $N>0$, our detection signals therefore give an upper bound for the actual state populations $p_{N}$.

Comparing the measured rotational state distribution to a Boltzmann distribution that gives the same ground-state population $p_{0}$ results in an effective rotational temperature $T_{\text {eff }}=26.5 \mathrm{~K}$. Although such a temperature could also be achieved using a cryogenic set-up, the all-optical method has several advantages: it puts less constraints on the design of the experiment, is faster than waiting for thermalization with a cold environment and compared with cryogenic buffer-gas cooling it does not compromise the translational temperature of the sample.

A logical next step is to extend the rotational cooling by a $1.3 \mathrm{THz}$ radiation source for addressing individual hyperfine components of the fundamental rotational transition $(0,0) \rightarrow(0,1)$. This will enable preparation of the ions also in a specific hyperfine sublevel of the rovibrational ground state and precise measurements of the
$\mathrm{HD}^{+}$hyperfine structure as well as improved absolute frequency measurements of rovibrational transition frequencies ${ }^{17,27,28}$.

\section{Methods}

Rate equation model. The rate equations for heteronuclear diatomics ${ }^{21}$ are based on Einstein $A$ and $B$ coefficients, which for $\mathrm{HD}^{+}$are derived from theoretical values for transition energies ${ }^{27}$ and transition dipole moments ${ }^{29}$. The numerical model includes states $(v=0-4, N=0-9)$ and interaction with the unpolarized blackbody radiation field as well as the linearly polarized laser fields for optical pumping and molecule detection. Rotational blackbody excitation and deexcitation is taken into account only in the $v=0$ level. The neglect of such processes within $v=1,2$ compared with vibrational spontaneous emission to the $v-1$ level requires that the ratio of the fundamental rotational frequency $v_{\text {rot }}$ and the blackbody temperature be sufficiently large, $\exp \left(h v_{\text {rot }} / k_{\mathrm{B}} T\right)-1 \gg 2 A_{11,10} / A_{11,00}$, where $A_{v^{\prime} N^{\prime}, v N}$ stands for the spontaneous decay rate from an upper level $\left(v^{\prime}, N^{\prime}\right)$ to a lower level $(v, N)$. For the relevant cases $T \leq 300 \mathrm{~K}$, this inequality is satisfied for most diatomics. Vibrational blackbody excitation and deexcitation is taken into account for all levels considered. It is negligible in $\mathrm{HD}^{+}$at room temperature compared with competing rates, but more generally may become relevant for the $(0,0)$ ground state, because the blackbody excitation rate $\Gamma_{00,11}(T)$ for $(0,0) \rightarrow(1,1)$ can become comparable to or larger than the rotational blackbody rate $\Gamma_{0}(T)$ for $(0,0) \rightarrow(0,1)$ at more elevated temperatures.

The main simplification of the model is the way we account for the detailed hyperfine structure of the rovibrational states. If the frequency spectrum of a radiation field driving a transition is broad compared with the spectral width of the transition's hyperfine spectrum (typically $<150 \mathrm{MHz}$ (ref. 28)), the hyperfine absorption spectrum can be approximated by a single line with a spectral profile given by the envelope of the actual hyperfine spectrum. As under typical experimental conditions the Doppler broadening of $10-20 \mathrm{MHz}$ of the hyperfine lines is comparable to the typical separation of adjacent hyperfine lines, we approximate each hyperfine spectrum by a single Gaussian absorption profile of appropriate width. For optical pumping to be efficient, the lasers must have similar spectral widths as the respective hyperfine spectra and sufficient spectral intensity to saturate the transitions.

Trapping and cooling apparatus. For translational cooling of the $\mathrm{HD}^{+}$ions, we simultaneously store both $\mathrm{Be}^{+}$and molecular ions in a linear radiofrequency trap driven at $14.2 \mathrm{MHz}$ with a peak-to-peak amplitude of $380 \mathrm{~V}$. The $\mathrm{Be}^{+}$ions are laser cooled on the ${ }^{2} S_{1 / 2}-{ }^{2} P_{3 / 2}$ transition at $313 \mathrm{~nm}$ down to temperatures of $<20 \mathrm{mK}$ (depending on the size of the ensemble) at which they form an ordered structure known as a Wigner or Coulomb crystal. Owing to the long-range electrostatic interaction between atomic coolants and molecular ions, the latter are sympathetically cooled to translational temperatures $<50 \mathrm{mK}$ and embedded in the $\mathrm{Be}^{+}$ion crystals in the vicinity of the trap symmetry axis. The ion ensembles have lifetimes ranging from minutes to hours, limited by chemical reactions with residual gas. The trapped species are identified by excitation of their mass-dependent motional (secular) frequencies. We excite the radial motion of the $\mathrm{HD}^{+}$ions by a spatially homogeneous and temporally oscillating electric field, resonant with the $\mathrm{HD}^{+}$radial secular frequency in the trap $(800 \mathrm{kHz})$, which is slightly shifted with respect to the single ion motional frequency $(840 \mathrm{kHz})$ because of the space charge of the ion ensemble. We use molecular dynamics simulations to deduce ion numbers, three-dimensional spatial distributions and upper limits for the translational temperature of each species contained in the crystal from CCD (charge-coupled device) camera images of the ion ensembles. The simulations include full Coulomb interaction, light pressure forces, anisotropies of the trap pseudopotential and species-dependent heating rates ${ }^{30}$.

Laser sources for optical pumping. For optical pumping, we use two laser sources: a room-temperature continuous-wave quantum cascade laser driving the

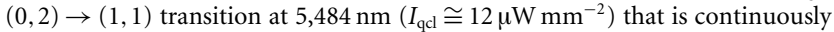
frequency referenced to an absorption line in $\mathrm{NH}_{3}$ and spectrally broadened to $100 \mathrm{MHz}$ (peak to peak). The second laser is a continuous-wave distributed feedback diode laser driving the $(0,1) \rightarrow(2,0)$ transition at $2,713 \mathrm{~nm}\left(I_{\mathrm{dfb}} \cong 0.3 \mathrm{~mW} \mathrm{~mm}^{-2}\right)$. During optical pumping, the centre frequency of the laser is not actively stabilized and the frequency spectrum is broadened to $200 \mathrm{MHz}$ (peak to peak) by current modulation. The rest of the time, the modulation is reduced to $40 \mathrm{MHz}$ (peak to peak) and the laser is continuously frequency referenced to an absorption line in $\mathrm{CO}_{2}$. For both the quantum cascade laser and the distributed feedback diode laser, the intensity is larger than the saturation intensity of the respective transition.

Received 1 September 2009; accepted 27 January 2010; published online 7 March 2010

\section{References}

1. Bethlem, H. L. \& Meijer, G. Production and application of translationally cold molecules. Int. Rev. Phys. Chem. 22, 73-128 (2003).

2. Rieger, T., Junglen, T., Rangwala, S. A., Pinkse, P. W. H. \& Rempe, G. Continuous loading of an electrostatic trap for polar molecules. Phys. Rev. Lett. 173002 (2005). 
3. Campbell, W. C. \& Doyle, J. M. in Cold Molecules (eds Krems, R. V., Stwalley, W. C. \& Friedrich, B.) 473-508 (CRC Press, 2009).

4. Fioretti, A. et al. Formation of cold $\mathrm{Cs}_{2}$ molecules through photoassociation. Phys. Rev. Lett. 80, 4402-4405 (1998).

5. Hodby, E. et al. Production efficiency of ultracold Feshbach molecules in bosonic and fermionic systems. Phys. Rev. Lett. 94, 120402 (2005).

6. Jochim, S. et al. Bose-Einstein condensation of molecules. Science 302, 2101-2103 (2003).

7. Regal, C. A., Ticknor, C., Bohn, J. L. \& Jin, D. S. Creation of ultracold molecules from a Fermi gas of atoms. Nature 424, 47-50 (2003).

8. Weinstein, J. D., deCarvalho, R., Guilett, T., Friedrich, B. \& Doyle, J. D. Magnetic trapping of calcium monohydride molecules at millikelvin temperatures. Nature 395, 148-150 (1998).

9. Mølhave, K. \& Drewsen, M. Formation of translationally cold $\mathrm{MgH}^{+}$and $\mathrm{MgD}^{+}$molecules in an ion trap. Phys. Rev. A 62, $011401(\mathrm{R})$.

10. Roth, B., Koelemeij, J. C. J., Daerr, H. \& Schiller, S. Rovibrational spectroscopy of trapped molecular hydrogen ions at millikelvin temperatures. Phys. Rev. A 74, 040501(R) (2006).

11. Offenberg, D., Zhang, C. B., Wellers, Ch., Roth, B. \& Schiller, S. Translational cooling and storage of protonated proteins in an ion trap at subkelvin temperatures. Phys. Rev. A 78, 061401(R) (2008).

12. Danzl, J. G. et al. Quantum gas of deeply bound ground state molecules. Science 321, 1062-1066 (2008).

13. Deiglmayr, J. et al. Formation of ultracold polar molecules in the rovibrational ground state. Phys. Rev. Lett. 101, 133004 (2008).

14. Lang, F., Winkler, K., Strauss, C., Grimm, R. \& Denschlag, J. H. Ultracold triplet molecules in the rovibrational ground state. Phys. Rev. Lett. 101, 133005 (2008)

15. Ni, K.-K. et al. A high phase-space-density gas of polar molecules. Science 322, 231-235 (2009).

16. Schiller, S. \& Korobov, V. Tests of time independence of the electron and nuclear masses with ultracold molecules. Phys. Rev. A 71, 032505 (2005).

17. Koelemeij, J. C. J., Roth, B., Wicht, A., Ernsting, I. \& Schiller, S. Vibrational spectroscopy of $\mathrm{HD}^{+}$with 2 -ppb accuracy. Phys. Rev. Lett. 98, 173002 (2007)

18. Krems, R. V., Stwalley, W. C. \& Friedrich, B. (eds) in Cold Molecules (CRC Press, 2009).

19. Morigi, G., Pinkse, P. W. H., Kowalewski, M. \& de Vivie-Riedle, R. Cavity cooling of internal molecular motion. Phys. Rev. Lett. 99, 073001 (2007).

20. Hudson, E. R. Method for producing ultracold molecular ions. Phys. Rev. A 79, 032716 (2009).

21. Vogelius, I. S., Madsen, L. B. \& Drewsen, M. Blackbody-radiation-assisted laser cooling of molecular ions. Phys. Rev. Lett. 89, 173003 (2002).
22. Højbjerre, K., Hansen, A. K., Skyt, P. S., Staanum, P. F. \& Drewsen, M. Rotational state resolved photodissociation spectroscopy of translationally and vibrationally cold $\mathrm{MgH}^{+}$ions: Toward rotational cooling of molecular ions. New J. Phys. 11, 055026 (2009).

23. Koelemeij, J. C. J., Roth, B. \& Schiller, S. Blackbody thermometry with cold molecular ions and application to ion-based frequency standards. Phys. Rev. A 76, 023413 (2007).

24. Vogelius, I. S., Madsen, L. B. \& Drewsen, M. Rotational cooling of heteronuclear molecular ions with ${ }^{1} \Sigma,{ }^{2} \Sigma, \Sigma$, and ${ }^{1} \Pi$ electronic ground states. Phys. Rev. A 70, 053412 (2004).

25. Morigi, G. \& Eschner, J. Doppler cooling of a Coulomb crystal. Phys. Rev. A 64, 063407 (2001).

26. Wineland, D. J. \& Itano, W. M. Laser cooling of atoms. Phys. Rev. A 20, 1521-1540 (1979).

27. Korobov, V. I. Leading-order relativistic and radiative corrections to the rovibrational spectrum of $\mathrm{H}_{2}^{+}$and $\mathrm{HD}^{+}$. Phys. Rev. A 74, 052506 (2006).

28. Bakalov, D., Korobov, V. I. \& Schiller, S. High-precision calculation of the hyperfine structure of the $\mathrm{HD}^{+}$ion. Phys. Rev. Lett. 97, 243001 (2006).

29. Colbourn, E. A. \& Bunker, P. R. Accurate theoretical vibration-rotation energies and transition moments for $\mathrm{HD}^{+}, \mathrm{HT}^{+}$, and $\mathrm{DT}^{+}$. J. Mol. Spectrosc. 63, 155-163 (1976)

30. Zhang, C.-B., Offenberg, D, Roth, B., Wilson, M. A. \& Schiller, S. Molecular-dynamics simulations of cold-single species and multispecies ion ensembles in a linear Paul trap. Phys. Rev. A 76, 012719 (2007).

\section{Acknowledgements}

The authors thank M. Hansen, S. Vasilyev, E. Zlobina for contributions to the experimental set-up and A. Nevsky for helpful discussions. This work is supported by the Deutsche Forschungsgemeinschaft under grant SCHI 431/11-1.

\section{Author contributions}

T.S. and B.R. planned and carried out the experiments, S.S. and H.D. participated, T.S. carried out data evaluation and numerical simulations, B.R. participated, S.S. and H.D. developed the quantum cascade laser and distributed feedback diode laser system, S.S. conceived the study, developed the rate equation model and analytic treatment, I.E. developed a near-infrared diode laser system and participated in preliminary and complementary investigations; T.S. wrote the paper, B.R. and S.S. participated.

\section{Additional information}

The authors declare no competing financial interests. Supplementary information accompanies this paper on www.nature.com/naturephysics. Reprints and permissions information is available online at http://npg.nature.com/reprintsandpermissions. Correspondence and requests for materials should be addressed to T.S. or S.S. 